\title{
Diabetic Autonomic Neuropathy
}

National Cancer Institute

\section{Source}

National Cancer Institute. Diabetic Autonomic Neuropathy. NCI Thesaurus. Code C27068.

Autonomic neuropathy that is caused by diabetes mellitus. 